www. revis tad y o. com

\title{
Factores que afectan la relación entre diversificación y rendimiento. Revisión de la literatura
}

\author{
Factors affecting the relationship between diversification and performance. Literature Review
}

\author{
Omar Alexánder León García ${ }^{1,2}$, Juan Ignacio Igartua López² y Jaione Ganzaraín Epalde² \\ ${ }^{1}$ Grupo de Investigación de Innovación y emprendimiento. Escuela Politécnica Superior -Mondragón Unibertsitatea \\ ${ }^{2}$ Facultad de Ingeniería Compensar - Unipanamericana Fundación Univeristaria. \\ omar.leon@alumni.mondragon.edu, jigartua@mondragon.edu, jganzarain@mondragon.edu
}

Fecha de recepción: 20-05-2015

Fecha de aceptación: 21-10-2015

Resumen: La relación entre la estrategia de diversificación y el rendimiento empresarial ha sido una temática de mucha importancia para académicos de diferentes áreas desde hace varias décadas, los cuales han reconocido diferentes factores que pueden afectar esta relación. La revisión de la literatura en la que se basa este artículo ha permitido identificar 11 factores principales que pueden impactar los resultados empresariales de una empresa que ha decidido diversificarse (Tipo, modo, grado, gobernanza, I+D, Tecnologías de la información y comunicación, riesgo, conocimiento, industria, tamaño y edad). El análisis se ha enmarcado en la evidencia empírica hallada en 55 estudios realizados durante el periodo comprendido entre 2003 y 2014.

Palabras clave: Diversificación, factores, rendimiento, teorías de diversificación, medición de la diversificación.

\begin{abstract}
The relationship between diversification and firm performance has been a topic of great importance to scholars of different areas for several decades and who have recognized some factors that can affect this relationship. A review of the literature on which this article is based has identified 11 key factors that can impact the business performance of a company that has decided to diversify (Type, mode, diversification level, governance, R\&D, Information and communication technologies, business risk, knowledge, industrial sector, size and age). The analysis has been based on empirical evidence found in 55 studies conducted during the period between 2003 and 2014.
\end{abstract}

Keywords: Diversification, factors, performance, theories of diversification, diversification measurement.

\section{Introducción}

Debido al rápido desarrollo de la economía global, muchas empresas optan por la diversificación como su elección estratégica (Song and Wang, 2011), además que la incorporación de actividades económicas nuevas se ha convertido en un hecho indispensable para transformarse o seguir siendo una empresa dinámica y con futuro (Benito 2009). Por esta razón, la diversificación ha llamado la atención del mundo empresarial así como de numerosos investigadores de diferentes áreas durante las últimas décadas, que buscan identificar los factores determinantes que la afectan y demostrar sus efectos sobre el desempeño empresarial basándose sobre diferentes teorías y conceptos.

Son varios los tipos de diversificación que puede aplicar una organización, ya sea porque el nuevo negocio al que se desea ingresar está relacionado, o no, con el negocio original de la empresa, o porque se encuentre en diferentes áreas geográficas, o de acuerdo al modo de entrada, ya sea por un desarrollo interno, adquisición o alianzas estratégicas (Chiang 2010). El impacto que tienen estos elementos se evidencia en la literatura, identificando cómo cada uno de estos puede afectar al rendimiento de la empresa diversificada, tal como la elección del modo de entrada (Busija et al., 1997; Tihanyi et al., 2005; de Souza, 2009), el tipo y grado de diversificación (Rumelt, 1982; Singh \& Montgomery, 1987; Pandya \& Rao, 1998; Gary, 2005; Chari et al., 2008; Marinelli 2011; Yang, Zhao et al., 2012; Villasalero 2013; Purkayastha 2013), la dirección, (Rawley and Simcoe, 2008) o la entrada en una diversificación internacional (Fletcher and Marshall 2005; Gaur and Kumar, 2009; Avdulaj and Barunik, 2013).

Este documento se divide en cuatro apartados. En el 
primero se evidencia la metodología utilizada para la selección de estudios del área de interés. El segundo trata sobre el marco de referencia que incluye la clasificación de los estudios, los tipos de medida de diversificación utilizados y las principales teorías en las que se basan las investigaciones. El tercer apartado muestra los factores que afectan el rendimiento de las empresas diversificadas obtenidos del análisis de los estudios y en la última parte se presenta una discusión a la literatura junto con las conclusiones en las que se identifican posibles líneas de investigación futuras.

\section{Metodología}

La metodología de análisis ha requerido de una selección sistemática de los artículos que se han ajustado a los objetivos y a la pregunta de investigación. La búsqueda de trabajos se centró en aquellos estudios que directamente relacionan la diversificación con el rendimiento empresarial, con la idea de identificar los factores determinantes que llevan a las empresas a diversificarse. Para esta búsqueda fueron utilizadas diferentes bases de datos como Scopus, Engineering Village, Science direct, IEEE Xplore, EBSCO y Wiley Springer.

Para hacer frente a esta cuestión sobre diversificación, se ha seguido el desarrollo de la literatura desde los trabajos seminales de (Ansoff, 1958), (Rumelt, 1974) y (Ramanujam and Varadarajan, 1989), que permitían identificar conceptos, términos y marcos para clasificar los tipos de estudios que se pudieran encontrar y permitieran identificar los términos clave de búsqueda, "diversification", "performance", "empirical", los cuales fueron igualmente argumentados y complementados a través de los estudios examinados. Así mismo se agregó el término "multibusiness" y "conglomerate" con el cual se pudiera abarcar otros estudios en los que se reconociera la diversificación empresarial. Una vez identificados estos estudios se reconocieron las revistas que presentaban mayor producción literaria del tema de estudio (por ejemplo Strategic Management Journal, Research Policy, European Management Journal, Academy of Management Journal, Corporate Governance, Journal of Applied Business Research, Organization Science, British Journal of Management, Corporate Ownership and Control).

Se procedió a seleccionar y extraer la información más relevante, con el propósito de descartar toda la que no fuera necesaria, y así reducir el volumen de los documentos que se iban a tratar. De esta manera fueron seleccionados 55 artículos que cumplían con dos criterios planteados. Por un lado debían incluir evidencia empírica, por tal razón se excluyeron aquellos que fueran revisiones teóricas de la literatura y capítulos de libro, y por otro lado, debían pertenecer al campo de la gestión estratégica, las ciencias de la decisión o la ingeniería. Estos campos se seleccionaron debido a los múltiples artículos relacionados a la temática identificados en ellos con un número significativo de citaciones.

Ya con los estudios seleccionados se realizó un análisis de sus planteamientos, revisando sus objetivos, hipótesis, factores de análisis y resultados, lo que permitió identificar los principales factores más utilizados y que han presentado mayor repercusión académica.

\section{Marco de referencia (Clasifi- cación de los estudios, medi- das y teorías de diversifi- cación)}

Debido al gran interés de académicos por el tema de la diversificación, se hace necesario clasificar las diferentes temáticas en las cuales se enmarcan dichos estudios. Así mismo se presenta a continuación una revisión de las medidas utilizadas y las principales teorías que abarcan estas investigaciones.

\subsection{Clasificación de los estudios}

Los estudios científicos seleccionados se podrían agrupar en un total de 11 temáticas: el entorno genérico, el entorno industrial o estructura del mercado, las características de la empresa, los motivos para diversificar, la elección de la dirección de la diversificación, el estado de diversidad alcanzado, la estructura de la diversidad, los procesos internos y sistemas, las sinergias, la rentabilidad ex-ante y la rentabilidad ex-post (Ramanujam and Varadarajan 1989). Inclusive, se puede reducir a 3 grandes grupos relacionados con la industria y la empresa, temas relacionados con la implantación y elección de la estrategia, y los temas relacionados con el rendimiento antes y después de implementar la diversificación. (Cortés et al., 2005).

Para este caso, la revisión abarca los tres grandes grupos en la medida que cada uno contiene diferentes factores que impactan tanto al rendimiento de la empresa como a los motivos, tipo y modo de entrada de la diversificación.

Se reconocen varios estudios que tratan sobre los motivos que llevan a la empresa a tomar la decisión de diversificarse basados en su mayoría desde la teoría de los recursos y capacidades, (Chatterjee and Wernerfelt, 1991; Nayyar, 1992; Gary, 2005; Miller, 2006; 
Forcadell et al., 2006; Fernández and Díez Vial, 2013). Dentro de estos motivos existen otros que desde la teoría de la agencia afectan a la gobernanza interna y a los diferentes elementos de la diversificación como el tipo y el modo de entrada y cómo ésta igualmente puede afectar a la estructura de gobierno de una empresa (Herrmann \& Datta, 2005; White et al., 2008; R. Chen et al., 2009; Chung \& Luo, 2008b; Singh \& Gaur, 2009).

Igualmente, estudios más recientes relacionan el tipo y grado de diversificación con los resultados económicos de la empresa (Gary, 2005; Miller, 2006; Alonso and Forcadell, 2010; Villasalero, 2013); (Tanriverdi, 2006; Chari, Devaraj et al., 2008; Bausch and Pils 2009; Ibrahim et al., 2009; Singh and Gaur, 2009; Park and Jang, 2012; O’Brien et al., 2014; Purkayastha, 2013), basándose en una serie de medidas continuas y categóricas de diversificación que indican cuanto están relacionados los negocios de la organización, (Montgomery, 1982; Palepu, 1985; Nayyar, 1992; Hoskisson, Hitt et al., 1993).

Otros estudios determinan la relación entre el tipo de diversificación y el modo de entrada en nuevos negocios, revelando en su mayoría una dependencia de elección entre uno y otro, (Kim and Hwang, 1992; Busija et al., 1997, Chang and Singh, 1999; de Souza Vilas, 2009; Sánchez and Menguzzato, 2009; Banker et al., 2011; Arikan and Stulz, 2011), siendo el desarrollo interno, la adquisición y las alianzas estratégicas las elecciones de entrada más comunes.

\subsection{Medida de la diversificación}

Diversificación significa la entrada de una empresa en nuevas líneas de productos, procesos, servicios o mercados (Steiner, 1964), que implican cambios en su estructura administrativa, el sistema y otros procesos de gestión (Ramanujam and Varadarajan 1989). Igualmente, Lichtenthaler (2005), la define como el incremento de los productos y mercados de una empresa. Según la literatura, la entrada en nuevas líneas de negocio está siempre relacionada con el rendimiento empresarial, lo que ha hecho imprescindible el uso de una medida de diversificación que permitiera comprobar los resultados encontrados (Villalonga, 2004; Tanriverdi, 2006; Miller 2006; Ravichandran et al., 2009; Sánchez and Menguzzato, 2009; Chen et al., 2009; Hai and Fu, 2011; Michael, 2007).

La medida de diversificación se refiere al grado o nivel en el cual una empresa opera entre diversos segmentos de negocio (Park and Jang, 2013). En general existen dos tipos de medida, las continuas y las categóricas. Dentro de las medidas continuas se destaca la contabilización de productos de la firma de acuerdo al porcentaje de ventas de sus productos en los diferentes mercados y es medido según la utilización de 2, 3 o 4 dígitos del SIC (Montgomery, 1982). Palepu (1985), muestra un índice de entropía, el cual identifica un índice de diversificación total y otros dos para distinguir entre diversificación relacionada y no relacionada. Este índice tiene una alta validez dentro de la medida de diversificación como lo indica Hoskisson et al., (1993), y se evidencia su utilización en algunos estudios (Tanriverdi, 2006; Ravichandran et al., 2009, Hai and Fu 2011, Wang and Yang, 2013).

Las medidas categóricas son de carácter cualitativo e identifican tanto el grado de diversificación como el tipo de relación existente entre los negocios. Esta clasificación se basa en los estudios de Wrigley (1970) y Rumelt (1974), que de acuerdo a una serie de ratios determinados por los porcentajes de venta de cada negocio, determina que la diversificación es relacionada cuando coexisten recursos compartidos entre los negocios en los que está involucrada la empresa y es no relacionada cuando se presenta un grado mayor de diferencia entre la situación actual de la empresa y los nuevos negocios.

Por otro lado, existe una medida de diversificación internacional, la cual se utiliza para medir la diversificación de mercados geográficos de la empresa tanto fuera como dentro del país (Garcia et al., 2003). En general consiste en una medida que relaciona las ventas internacionales con el porcentaje de las ventas totales de la empresa, asignándoles un peso a cada mercado (Hitt et al., 1997). Se puede evidenciar este tipo de diversificación y medida en varios estudios (Herrmann and Datta, 2005; Wiersema and Bowen, 2008; Gaur and Kumar, 2009; Ibrahim et al., 2009; Fernández and Díez, 2013; Hauser et al., 2013, Kang, 2013; Sanchez and Usero, 2013).

\subsection{Teorías conceptuales de la decisión de diversificarse}

Las razones por las cuales una empresa se diversifica han definido diferentes aspectos que tratan de dar respuesta a la pregunta de por qué una empresa decide diversificarse (Montgomery, 1994). La teoría de la agencia, la visión de mercados, la de los recursos, las capacidades dinámicas, la teoría del conocimiento y la teoría de costes de transacción, son algunas de estas perspectivas, en las cuales se han enmarcado los diferentes estudios de diversificación, y que evidencian la mayoría de las bases de los estudios empíricos como lo indica la tabla 1. Mientras las dos primeras se caracterizan por ser del campo económico, las otras tres estarían dentro del área de la dirección estratégica (Ríos, 2012). 
Tabla 1. Teorías sobre la decisión de diversificación de las empresas

\begin{tabular}{|c|c|c|}
\hline Teoría & Características & Referencias \\
\hline Teoría de los recursos & $\begin{array}{l}\text { Ingreso en nuevos negocios por exceso de } \\
\text { recursos. } \\
\text { Generar sinergias }\end{array}$ & $\begin{array}{l}\text { Gary (2005), Miller (2006), Forcadell et } \\
\text { al., (2006), Tanriverdi (2006), Alonso and } \\
\text { Forcadell (2010), Fernández and Díez } \\
\text { (2013). }\end{array}$ \\
\hline Teoría de capacidades dinámicas & $\begin{array}{l}\text { Creación y captura de riqueza. Capacidades } \\
\text { como ventaja competitiva }\end{array}$ & $\begin{array}{l}\text { Alonso and Forcadell (2010), Liu and Hsu } \\
\text { (2011), Sambharya and Lee (2014) }\end{array}$ \\
\hline Teoría de poder de mercado & Innovación y entrada en nuevos mercados & Bausch and Pils (2009), White et al., (2008) \\
\hline Teoría de la agencia & $\begin{array}{l}\text { Aspectos discrecionales } \\
\text { Interés de directivos y administradores }\end{array}$ & $\begin{array}{l}\text { Chung and Luo (2008), Singh and Gaur } \\
\text { (2009), Mendes-da-Silva et al., (2008) }\end{array}$ \\
\hline Teoría de costes & Costes organizacionales & $\begin{array}{l}\text { Gary (2005), Sánchez and Menguzzato } \\
\text { (2009), O'Brien et al., (2014), Mishra and Ak- } \\
\text { bar (2007) }\end{array}$ \\
\hline Teoría del conocimiento & $\begin{array}{l}\text { Gestión del conocimiento, barreras y costos } \\
\text { de entrada, flujo de conocimiento }\end{array}$ & $\begin{array}{l}\text { Miller et al., (2007), Tanriverdi (2005), Villa- } \\
\text { salero (2013) }\end{array}$ \\
\hline
\end{tabular}

Benito et al., (2012), indican que en la últimas décadas la teoría de los recursos ha sido una de las que más influencia ha tenido sobre los estudios que relacionan la diversificación y los resultados empresariales. Este punto de vista sugiere que el tipo de estrategia de diversificación que adopte una empresa y su rendimiento están condicionados por el conjunto de recursos y capacidades con que cuente.

Cada una de estas teorías, ha tenido una importante trascendencia en las diferentes décadas de estudios de la diversificación, ya que han sido los pilares en los que se basa cada uno de los planteamientos realizados por los autores, siendo la teoría de recursos y capacidades la que más se evidencia en los estudios bajo la premisa de que el exceso de recursos es la principal razón por la cual las empresas deciden entrar en una estrategia de diversificación. Mientras la teoría de la agencia soporta los estudios de gobernanza y estructura organizacional de las empresas diversificadas.

\section{Factores que afectan la rela- ción entre diversificación y rendimiento empresarial}

El rendimiento de la empresa que decide entrar en nuevas líneas de negocio puede verse afectada por diferentes razones que varían de acuerdo a los aspectos relacionados con su implementación, las decisiones estratégicas que tome, las características internas de la empresa, así como los aspectos externos de la industria en la que se encuentra.

Dentro de los aspectos relacionados con la implementación se han identificado como factores clave el tipo y modo de entrada elegidos en las nuevas líneas de negocio. Los factores estratégicos tales como la gobernanza, la apuesta en investigación y desarrollo que hace la empresa $(I+D)$, la inversión en tecnologías de información y comunicación (TIC), la gestión del conocimiento y el riesgo empresarial, son identificados como determinantes de la estrategia de diversificación y moderadores de su relación con el rendimiento empresarial.

A nivel externo se incluye la situación macroeconómi$\mathrm{ca}$, entendida como el comportamiento industrial del sector de análisis, su concentración, crecimiento, características del país etc., los cuales son un factor fundamental de análisis tanto de implementación de la diversificación como del rendimiento empresarial. Ver figura 1. 


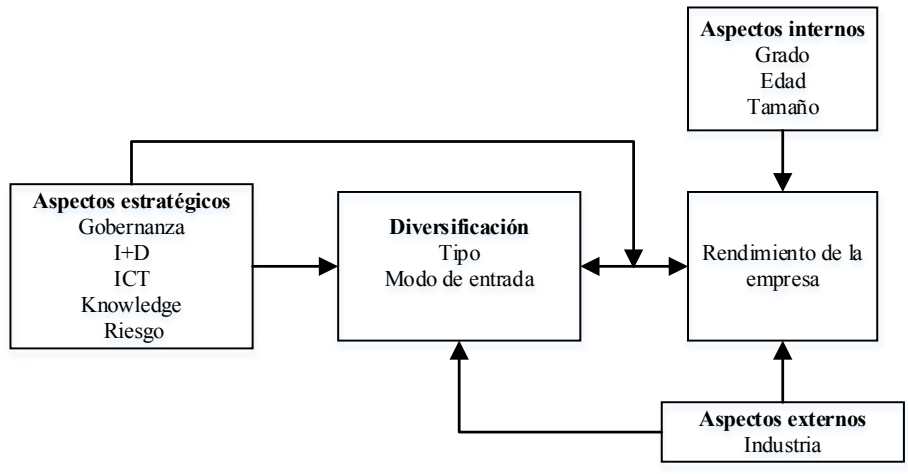

Figura 1. Factores que afectan la relación entre diversificación y rendimiento empresarial.

Se observa también que la relación entre la diversificación y el rendimiento empresarial se da en ambos sentidos, con lo cual se entiende que los resultados empresariales pueden verse impactados por la implementación de una nueva línea de negocio, así como el rendimiento puede afectar el tipo y modo de entrada en un nuevo proceso de diversificación. Además, de acuerdo a la literatura, se identifican como factores internos los aspectos referentes a la edad, el tamaño y el grado de diversificación que tiene la empresa.

A continuación se analizan las principales características de cada uno de los aspectos identificados.

\subsection{Aspectos de la diversificación}

\section{a. Tipo de diversificación}

Se asume que la diversificación relacionada se basa en extraer los beneficios de la utilización sinérgica de recursos y capacidades en nuevos campos de actividad (Gary, 2005), mientras que la diversificación no relacionada hace referencia a que el nuevo campo de actividad no produce ninguna similitud con las antiguas áreas de negocio, productos y recursos tecnológicos.

El trabajo de Rumelt (1974), quien adoptó una medida categórica de la diversificación, fue pionero entre los estudios de gestión estratégica que examinaron sus beneficios en el rendimiento, diferenciando los resultados según el tipo de diversificación, siendo superior para la diversificación relacionada sobre la no relacionada. Estos hallazgos fueron confirmados más adelante por Christensen and Montgomery (1981). A esto se suma que las empresas que persiguen la diversificación relacionada durante un período prolongado de tiempo, logran un rendimiento significativamente superior, y que las empresas con un conjunto de negocios no relacionados no logran alcanzar este mismo rendimiento (Palepu, 1985).
Dentro de los estudios más recientes hay varios que proporcionan soporte a la ventaja de la diversificación relacionada sobre la no relacionada en cuanto a su impacto sobre los resultados empresariales (Tanriverdi and Venkatraman, 2005; Miller, 2006; Bausch and Pils, 2009; Villasalero, 2013; Park and Jang, 2013). A su vez, para otros autores las empresas con diversificación no relacionada supera en el rendimiento a las empresas con diversificación relacionada (Varadarajan, 1986; Kuppuswamy and Villalonga, 2010).

Por otro lado, existe una jerarquía basada en las posibles combinaciones de tipos de diversificación y la elección del modo de entrada que pueden afectar al rendimiento, encontrando un mejor ajuste entre la diversificación relacionada y el desarrollo interno, que entre la diversificación no relacionada y el desarrollo externo (Simmonds, 1990). Para Sánchez et al., (2010), el tipo de diversificación puede ser determinante en el modo de entrada de acuerdo al grado de diversificación y a las características de la industria.

\section{b. Modo de entrada}

Las empresas no siempre cuentan con las capacidades y competencias necesarias para ingresar en nuevos negocios, lo que lleva a considerar la opción de desarrollarlas internamente, adquirirlas por medio de otra empresa o hacer alianzas de cooperación con otras firmas (Chatterjee and Singh, 1999; Lichtenthaler, 2005). Desde la teoría de los recursos, la elección del modo de entrada se da en la medida que ésta puede implicar la necesidad de desarrollar nuevos recursos y capacidades (Andreu et al., 2010), mientras que con respecto al mercado, (Arikan and Stulz, 2011) indica que éste reacciona de manera más favorable a las adquisiciones por parte de empresas jóvenes que a las adquisiciones por parte de empresas maduras.

Sánchez and Menguzzato (2009), indican que las investigaciones en este campo se han centrado exclusivamente en el desarrollo interno y adquisiciones como forma de entrar en nuevos negocios y dejan de lado el modo híbrido que podrían dar las alianzas estratégicas. Según el estudio de (Barney, 1988), el tipo y el modo de diversificación tienen una estrecha relación, ya que, como sugiere la diversificación relacionada, favorece extender sus fortalezas a través del desarrollo interno, afectando positivamente el rendimiento. Al contrario, Lichtenthaler (2005), indica que la incidencia del modo de estrategia no varía a través de los diferentes tipos de estrategia, relacionada o no relacionada, si no de acuerdo a las competencias que tenga o deba adquirir la empresa. 


\subsection{Aspectos estratégicos}

\author{
a. Gobernanza
}

La estructura de gobierno tiene un efecto moderador en el rendimiento de la empresa diversificada (Hoskisson and Hitt, 1990), ya que según sea su estructura de familia, de instituciones inversionistas, gobiernos y demás, se da la correspondiente participación en la diversificación (George, 2007).

La revisión de la literatura empírica confirma que la estructura de propiedad tiene implicación sobre el rendimiento, analizándola desde diferentes puntos de vista, tales como la composición de la junta directiva y el tipo de diversificación (Chen et al., 2009), la propiedad e inversión en bancos (Chen and Wang, 2012), o el liderazgo y control de familia (Chung and Luo, 2008; Sanchez and Usero, 2013). Además de estas, Herrmann and Datta (2005), hacen un estudio sobre la importancia de las características del TMT (Top Managment Team), entre las que destacan los conocimientos técnicos y la experiencia profesional e internacional.

Igualmente, algunos estudios relacionan cómo se da la estructura de propiedad de una firma diversificada al pertenecer a un grupo de negocios (Khanna and Palepu, 2000; White et al., 2008; Lee and Jin, 2009), en cómo afecta el rendimiento en una diversificación internacional (Herrmann and Datta, 2005) y más sobre una diversificación relacionada que sobre una no relacionada (Chung and Luo, 2008).

$$
\text { b. I+D }
$$

Otro factor que afecta a la diversificación tiene que ver con la inversión y adopción del I+D. Dentro de los estudios revisados, Alonso and Forcadell (2010), analizan el desempeño del I+D en una empresa en un período de tiempo, identificando que, hasta cierto momento, dicho desempeño crece y después se nota un decrecimiento hasta que la empresa asume un proceso de diversificación no relacionada, siendo este comportamiento de $\mathrm{U}$ invertida característico de las empresas. Acorde con esto Forcadell et al., (2006), indicaban que el comportamiento de I+D es positivo con la diversificación relacionada pero que marginalmente con el tiempo decrece.

Chiang (2010), propone que el gasto en I+D sea más valorado dentro de empresas que diversifican en la misma industria. Así mismo la intensidad de I+D en empresas diversificadas esta negativamente relacionada con una medida continua de diversificación y, comparado con la diversificación relacionada, la no relacionada inhibe la inversión de I+D en la empresa
(Wang and Yang, 2013). Rodríguez et al., (2007), sugieren que la innovación conlleva a la diversificación, pero que un mayor grado de diversificación no necesariamente aumenta el nivel de innovación de la empresa. Finalmente, la literatura empírica proporciona apoyo a las teorías basadas en los recursos, lo que indica que las empresas con una inversión en I + D intensiva son más propensas a diversificar (Gourlay and Seaton, 2004).

$$
\text { c. TIC }
$$

Las investigaciones empíricas en su mayor parte relacionan la diversificación con las TIC en como su gasto o inversión complementa significativamente esta estrategia y está generalmente relacionada con los resultados empresariales (Michael, 2007; Ravichandran et al., 2009; Chari et al., 2008). De hecho, Ravichandran et al., (2009), especifican un mayor impacto de inversión en TIC en la diversificación relacionada que en la no relacionada.

De otro lado Sandulli et al., (2012), mide la adopción de $\mathrm{TIC}$ en las empresas y su impacto en la productividad de la organización. Para esto identifica el nivel de computarización de los que considera las tres principales áreas funcionales de la empresa: las operaciones, contabilidad y ventas y marketing, relacionando esta medida con el tipo de diversificación implementado en la empresa.

Shin, (2006), agrega que los estudios que relacionan las TIC y diversificación indican que las empresas diversificadas, especialmente en líneas relacionadas de negocio, hicieron mayores inversiones en TIC y que los hallazgos podrían reflejar una mayor necesidad de coordinación de los medios y procesamiento de la información dentro de las empresas diversificadas.

\section{d. Riesgo}

Uno de los elementos positivos de la diversificación es la reducción del riesgo de la empresa ya que participar con el mayor número de negocios implica una reducción en el mismo (Grant, 1998). Con reducir el riesgo de la firma se busca crear valor con estrategias como la de crear escudos fiscales de interés; con lo que se espera que las empresas multinegocio tengan una mayor influencia y que el pago de impuestos sea más bajo que sus negocios en caso de operar por separado, (Galván, 2009).

Desde otro punto de vista, para evitar la incertidumbre y el riesgo, las empresas tratan de mejorar las capacidades sobre la base de las ya existentes. La posibilidad de renovar y actualizar las capacidades potencial- 
mente más valiosos es un factor crítico de la estrategia de crecimiento basada en la capacidad, ya que estas actividades pueden alterar fundamentalmente la base de competencia de una empresa, haciendo más fácil buscar nuevas oportunidades en el mercado (Liu and Hsu, 2011). Así mismo, se identifica al riesgo como un factor fundamental que afecta a el rendimiento de la estrategia de diversificación de acuerdo al perfil que maneja cada empresa (Ibrahim et al., 2009, Cressy et al., 2012).

\section{e. Capacidades de conocimiento}

Desde el estudio de Barney (1991), se destaca la importancia de los activos intangibles, como el conocimiento, para la consecución de un rendimiento superior y una ventaja competitiva sostenible. De hecho, la ventaja competitiva de una empresa puede basarse en la eficacia con la que se aprovechan las capacidades de gestión del conocimiento en toda la cadena de suministro de la empresa (Collins et al., 2010), además de ser cultivo de innovación, mediante la búsqueda y la transferencia de conocimientos a través de las divisiones en una empresa diversificada (Miller et al., 2007).

Algunos de los estudios revisados, muestran cómo se impacta el rendimiento de la empresa diversificada con la transferencia de aprendizaje y conocimiento organizacional (Villasalero, 2013), o con las capacidades del equipo de gestión (Herrmann and Datta, 2005), mientras que (Leten et al., 2007) hacen un estudio del aporte del conocimiento y la diversificación tecnológica. Este estudio identifica la importancia en las empresas de la capacidad de conocimiento, considerando que las empresas pueden aumentar los beneficios de la diversificación y pueden limitar las desventajas por la elección de la dirección de la diversificación cuidadosamente y ampliar sus actividades en los campos de la tecnología que comparten una base común de conocimientos con la cartera de la tecnología existente de la empresa. Además de esto, la gestión del conocimiento es una capacidad crítica a través de la cual las TIC afectan el rendimiento financiero de la empresa. (Tanriverdi, 2005).

La gestión de conocimiento que hace la empresa es un factor determinante en el modo de entrada de la empresa, teniendo en cuenta elementos como la dificultad de crear, transferir y copiar el know-how entre unidades de negocio (Sánchez and Menguzzato, 2009).

\subsection{Aspectos internos}
a. Grado de diversificación

El grado de diversificación se convierte en una medida de la experiencia que tiene la empresa en operar en diferentes negocios (Sánchez and Menguzzato, 2009). Este grado ha sido fundamental en los estudios realizados en la última década ya que se evidencia su impacto sobre el rendimiento empresarial (Shi et al., 2010), así como en la elección del modo de entrada al iniciar un nuevo proceso de diversificación (Sánchez and Menguzzato, 2009).

Mientras que Palepu, (1985), consideraba inicialmente que no había relación entre la diversificación y el rendimiento empresarial, dentro de los estudios analizados se evidencia que algunos presentan una relación positiva en este aspecto de acuerdo al grado de diversificación de la organización (Miller, 2006; Villalonga, 2004; Kuppuswamy and Villalonga, 2010), mientras que para otros esta relación es negativa (Hoskisson et al., 1993; Bausch and Pils, 2009; Shi et al., 2010), más aún si esta relación se da desde el punto de vista de la teoría de la agencia (Song and Wang, 2011).

Existe igualmente una relación positiva entre el rendimiento de la empresa y el grado de diversificación internacional, (Gaur and Kumar, 2009). Acorde con esto Ravichandran et al., (2009), indica también una relación positiva bajo ciertos criterios de inversión en TIC que haga la empresa.

\section{b. Tamaño y edad de la empresa}

En la mayoría de estudios se evidencia que los factores tamaño y edad de la empresa son utilizados como variables de control (Nachum, 2004; Yiu, Bruton et al., 2005; MacKey and Barney, 2006; Gong, Wang et al., 2008; Tanriverdi and Lee, 2008; Gubbi, Aulakh et al., 2009; Singh and Gaur, 2009; Yildirim-Öktem and Üsdiken, 2010; Cressy, Malipiero et al., 2012). Sin embargo, hay que resaltar los aporte hechos por Shyu and Chen (2009), Arikan and Stulz (2011) y Ríos (2012) que relacionan la importancia del ciclo de vida de la firma y su impacto directo sobre el rendimiento.

En general, las empresas que diversifican en sus etapas iniciales de crecimiento experimentan un efecto negativo importante en el rendimiento, diferente a cuando se hace en una etapa madura (Shyu and Chen, 2009). 
Sorprendentemente, las empresas diversifican temprano en su ciclo de vida y hay una probabilidad media de que la primera adquisición que hacen las empresas, es una adquisición de diversificación (Arikan and Stulz, 2011).

\subsection{Aspectos externos}

\author{
a. Industria
}

La industria juega un papel importante en el rendimiento de la empresa diversificada, ya que, aunque se evidencia un predominio de las empresas diversificadas frente a las especializadas, el rendimiento de la diversificación en todos los sectores no es homogéneo, ni positivo ni negativamente, ya que mientras las empresas diversificadas dominan algunos sectores de la industria, las especializadas lo hacen en otros (Santaló \& Becerra, 2006). Este factor es tenido en cuenta como una variable importante en los estudios empíricos, como lo hizo inicialmente Montgomery (1982), utilizando el índice de Herfindahl, para medir la concentración industrial, o Lien and Klein (2013), quien la valora según el índice de crecimiento industrial, entendido como el porcentaje de crecimiento de las ventas del sector industrial en un período de tiempo.

Los resultados empresariales se ven afectados de acuerdo al tipo de diversificación siempre y cuando se analice la industria en la cual está la organización, ya que esta puede ser positiva en una diversificación relacionada para una determinada industria o negativa para otra (Purkayastha, 2013).

En este último aspecto, se evidencia un vacío en los estudios sobre la importancia de la variable país (entorno institucional), y que puede ser fundamental en el impacto del sector industrial y por ende en los resultados económicos de las empresas diversificadas (Benito et al., 2012).

La tabla 2 resume los diferentes factores que se evidencian en los estudios empíricos de diversificación, así como las características que se tienen en cuenta en su medición, sus características y autores que las referencian.

Tabla 2. Factores que afectan el rendimiento de la empresa diversificada

\begin{tabular}{|c|c|c|}
\hline Factor & Características & Referencias \\
\hline \multirow[t]{2}{*}{ Tipo } & $\begin{array}{l}\text { Mayores beneficios de la diversificación relacion- } \\
\text { ada sobre la no relacionada }\end{array}$ & $\begin{array}{l}\text { Tanriverdi and Venkatraman (2005); Miller (2006); } \\
\text { Bausch and Pils (2009); Villasalero (2013). Park } \\
\text { and Jang (2013) }\end{array}$ \\
\hline & $\begin{array}{l}\text { Mayores beneficios de la diversificación No rela- } \\
\text { cionada sobre la relacionada }\end{array}$ & Kuppuswamy and Villalonga (2010). \\
\hline Modo de entrada & $\begin{array}{l}\text { Elección del modo de entrada (Desarrollo inter- } \\
\text { no, Adquisiciones, Cooperación) de acuerdo al } \\
\text { grado y tipo de diversificación }\end{array}$ & Sánchez et al., (2010) \\
\hline \multirow{3}{*}{ Gobernanza } & $\begin{array}{l}\text { Existe una relación positiva entre la diversifi- } \\
\text { cación total y la independencia de los directivos }\end{array}$ & Chen, Dyball et al., (2009) \\
\hline & $\begin{array}{l}\text { Empresas con un dominio de propiedad familiar } \\
\text { son menos propensos a desprenderse de nego- } \\
\text { cios no relacionados }\end{array}$ & Chung and Luo (2008) \\
\hline & $\begin{array}{l}\text { Empresas con mayores niveles de diversificación } \\
\text { internacional tienen un TMT con un mayor nivel } \\
\text { educativo, más jóvenes y con mayor experiencia }\end{array}$ & Herrmann and Datta (2005) \\
\hline $1+D$ & $\begin{array}{l}\text { La inversión que hacen las empresas en I+D } \\
\text { tiene un efecto positivo en la diversificación }\end{array}$ & $\begin{array}{l}\text { Miller (2006), Alonso and Forcadell (2010), } \\
\text { Chiang (2010), Leten et al., (2007), Michael } \\
\text { (2007), Sambharya and Lee (2014) }\end{array}$ \\
\hline
\end{tabular}




\begin{tabular}{|c|c|c|}
\hline \multirow{3}{*}{ TIC } & $\begin{array}{l}\text { El gasto que hace la empresa en TIC puede } \\
\text { complementar la estrategia de diversificación. } \\
\text { Existe una relación positiva entre la inversión en } \\
\text { TIC y rendimiento en la diversificación relacion- } \\
\text { ada }\end{array}$ & Michael (2007); Ravichandran, Liu et al., (2009) \\
\hline & $\begin{array}{l}\text { El rendimiento obtenido de las inversiones en } \\
\text { TIC es mayor en la diversificación relacionada } \\
\text { que en la diversificación no relacionada. }\end{array}$ & Chari, Devaraj et al., (2008) \\
\hline & $\begin{array}{l}\text { El uso intensivo de las TIC en los procesos de } \\
\text { operaciones se encuentra asociado con aumen- } \\
\text { tos sustanciales en la productividad de las em- } \\
\text { presas diversificadas. }\end{array}$ & Sandulli, et al., (2012) \\
\hline Riesgo & $\begin{array}{l}\text { Las empresas más diversificadas muestran un } \\
\text { mejor perfil de riesgo. }\end{array}$ & Ibrahim, et al., (2009) \\
\hline \multirow{2}{*}{ Capacidades de conocimiento } & $\begin{array}{l}\text { La relación del conocimiento y la innovación } \\
\text { tecnológica afecta el rendimiento de la empresa } \\
\text { diversificada. }\end{array}$ & $\begin{array}{l}\text { Tanriverdi and Venkatraman (2005); Leten, et al., } \\
\text { (2007), Villasalero (2013) }\end{array}$ \\
\hline & $\begin{array}{l}\text { Relación entre la dificultad de crear, transferir, } \\
\text { copiar y comprender know how entre unidades } \\
\text { de negocio y el modo de entrada }\end{array}$ & Sánchez and Menguzzato (2009) \\
\hline Industria & $\begin{array}{l}\text { Analiza el crecimiento y concentración industrial } \\
\text { como factor que puede afectar el rendimiento de } \\
\text { la empresa diversificada. }\end{array}$ & $\begin{array}{l}\text { Tanriverdi and Lee (2008), Wiersema and Bowen } \\
\text { (2008), Chiang (2010), Purkayastha (2013), Lien } \\
\text { and Klein (2013) }\end{array}$ \\
\hline Grado & $\begin{array}{l}\text { Relaciona el grado de diversificación y el ren- } \\
\text { dimiento empresarial }\end{array}$ & $\begin{array}{l}\text { Hoskisson et al., (1993), Villalonga (2004), Mill- } \\
\text { er (2006), Kuppuswamy and Villalonga (2010), } \\
\text { Bausch and Pils (2009), Shi et al., (2010), Song } \\
\text { and Wang (2011) }\end{array}$ \\
\hline Edad y ciclo de vida & $\begin{array}{l}\text { Relación entre la edad y el rendimiento de la em- } \\
\text { presa diversificada }\end{array}$ & $\begin{array}{l}\text { Herrmann and Datta (2005), Chung and Luo } \\
\text { (2008), Tanriverdi and Lee (2008), Chiang (2010), } \\
\text { Liu and Hsu (2011), Hauser et al., (2013), Shyu } \\
\text { and Chen (2009), Purkayastha (2013), Sanchez } \\
\text { and Usero (2013) }\end{array}$ \\
\hline Tamaño & $\begin{array}{l}\text { Relacionan número de empleados y ventas de la } \\
\text { empresa y su efecto como variable que afecta el } \\
\text { rendimiento. }\end{array}$ & $\begin{array}{l}\text { Chari et al., (2008), Banker et al., (2011), Samb- } \\
\text { harya and Lee (2014) }\end{array}$ \\
\hline
\end{tabular}




\section{Discusión y conclusiones}

Dada la revisión literaria realizada sobre la diversificación empresarial, se evidencia un amplio interés académico del área en cada uno de los factores que la afectan y de cómo ésta repercute en el rendimiento de la organización. Sin embargo, se ha identificado una teoría fragmentada sobre los determinantes que afectan esta relación y que justifica la necesidad de generar estudios que traten de integrar estos factores con el fin de reconocer de manera conjunta sus potenciales efectos.

Los resultados encontrados demuestran que en el rendimiento empresarial de una empresa diversificada impactan una serie de elementos de orden interno, externo y estratégico. Los dos primeros resultan ser factores necesarios y complementarios en los análisis empíricos que se realizan y que en su mayoría son considerados como variables de control, tales como el tamaño y edad de la empresa, así como las características de la industria, siendo justificados en cada uno de los estudios revisados.

En cuanto a los aspectos de gobernanza, la intensidad de I+D, la inversión en las TIC, el riesgo empresarial y las capacidades de conocimiento, poseen una amplia evidencia empírica y han generado una gran cantidad de literatura. Estos elementos de tipo estratégico son identificados de manera independiente en los análisis encontrados en la literatura.

De otro lado, es evidente que dentro de estos estudios se identifica que los factores que tienen también un alto interés académico, tiene que ver con el tipo y modo de entrada elegidos por la empresa que se diversifica, siendo las medidas categóricas de Wrigley (1970) y Rumelt (1974) las más utilizadas en los análisis del tipo de diversificación, mientras el desarrollo interno, adquisiciones y alianzas estratégicas las más adecuadas para el análisis del modo de entrada (Sanchez and Menguzzato, 2009).

Así mismo, las características ambientales del país de origen del negocio pueden tener diferencias importantes que pueden afectar el rendimiento empresarial y que es razonable tenerla en cuenta en la elección estratégica de la empresa (Benito et al., 2012), la cual es otra línea que necesita más estudios de investigación.

De otro lado se ha identificado que los principales marcos conceptuales en los que se desarrollan estos estudios pasan desde la teoría de la agencia, en su mayoría con los aportes en el estructura de capital y gobernanza, la teoría del conocimiento, con sus aportes sobre capacidades y habilidades humanas y de aprendizaje empresarial, y la teoría de recursos, que junto a la teoría de las capacidades dinámicas, enmarcan lo referente a la influencia de los recursos y capacidades de la empresa, las sinergias en las unidades de negocio, el impacto de las TIC, entre otros.

En algunos estudios se observa que la variedad de puntos de vista teóricos y métodos de investigación utilizados por los investigadores evidencia la diversidad de resultados encontrados, crítica que se ha hecho desde diferentes investigaciones realizadas a través del tiempo (Benito et al., 2012). Se evidencia igualmente, que para la mayoría de los académicos el uso de las medidas continuas de Palepu (1985) y Hoskisson et al., (1993), siguen siendo las de mayor utilización para identificar el grado de diversificación de las organizaciones..

Se identifica también que la mayoría de estos estudios dejan un poco descuidado los aspectos determinantes de la implementación de la estrategia, tales como la decisión que lleva a las empresas a diversificarse (Martínez and Fernández, 2009), y el modo de entrada que eligen (Andreu et al., 2010). Ya desde Lichtenthaler (2005) se reconoce una falta de investigación sobre cómo identificar y valorar las oportunidades de diversificación y que entradas son requeridas para la aplicación de esta estrategia, a las que Schuh et al., (2013) definen como las opciones que tiene una organización de diversificarse, siendo este un tema de atención para investigaciones futuras.

Finalmente, y de acuerdo a la literatura, las organizaciones maduras y en crecimiento desean diversificarse, ya sea porque creen tener cierta experiencia empresarial exitosa o porque sienten la capacidad de llevarla a nuevos retos en nuevas industrias y mercados alternos con la idea de mantener los mismos o mejores resultados de desempeño diversificándose (Shi et al., 2010). Sin embargo, a veces no tienen claro que factores deben tener en cuenta para su implementación, ya que un apoyo exclusivo sobre uno de los aspectos identificados puede resultar insuficiente para considerar tanto la entrada en la estrategia como su impacto en los resultados.

\section{Referencias}

ALONSO-BORREGO, C. and F. J. FORCADELL (2010). "Related diversification and R\&D intensity dynamics." Research Policy 39(4), pp. 537-548. 
ANDREU, R., E. CLAVER, and D. QUER (2010). "Entry of Spanish tourism firms into new businesses." International Journal of Contemporary Hospitality Management 22(1), pp. 7-23.

ANSOFF, H. I. (1958). "A model for diversification." Management Science 4(4), pp. 392-414.

ARIKAN, A. M. and R. M. STULZ (2011). "Corporate acquisitions, diversification, and the firm's lifecycle", National Bureau of Economic, (NBER Working Paper No. 17463) Ohio State University.

AVDULAJ, K. and J. BARUNIK (2013). "Can we still benefit from international diversification? the case of the Czech and German stock markets." Finance a Uver - Czech Journal of Economics and Finance. 63, p 5.

BANKER, R. D., S. WATTAL, and J. M. PLEHN-DUJOWICHET. (2011). "R\&D versus acquisitions: Role of diversification in the choice of innovation strategy by information technology firms." Journal of Management Information Systems 28(2), pp. 109-144.

BARNEY, J. (1991). "Firm resources and sustained competitive advantage." Journal of Management 17(1), pp. 99-120.

BARNEY, J. B. (1988). "Returns to bidding firms in mergers and acquisitions: Reconsidering the relatedness hypothesis." Strategic Management Journal 9(S1), pp. 71-78.

BASU, A. and S. MUYLLE (2011). "Assessing and enhancing e-business processes." Electronic Commerce Research and Applications 10(4), pp. 437499.

BAUSCH, A. and F. PILS (2009). "Product diversification strategy and financial performance: Meta-analytic evidence on causality and construct multidimensionality." Review of Managerial Science 3 (3), pp:157-190.

BENITO, O. D., M. L. Á. GUERRAS, and V. J. Á. ZUÑIGA (2012). "Four decades of research on product diversification: a literature review." Management Decision 50(2), pp. 325-344.

BENITO OSORIO, D. (2009). "Theoretical analysis of the relation between corporate diversification and performance." Análisis teórico de la relación entre diversificación corporativa y resultados empresariales 15(2), pp. 105-126.

BUSIJA, E. C., H. M. O'NEILL, and C. P. ZEITHAML (1997). "Diversification strategy, entry mode, and performance: Evidence of choice and constraints." Strategic Management Journal 18(4), pp. 321-327.

COLLINS, J. D., W. J. WORTHINGTON, P. M. REYES and M. ROMERO (2010). "Knowledge management, supply chain technologies, and firm performance." Management Research Review 33(10), pp. 947-960.

CORTÉS, E. C., R. A. GUERRERO, D. Q. RAMÓN (2005). "Modo de entrada en nuevos negocios de las empresas turísticas españolas: una explicación desde la teoría de recursos y capacidades." Revista de economía y empresa 23(54), pp. 129-150.

CRESSY, R., A. MALIPIERO, F. MUNARI (2012). "Does $V C$ fund diversification pay off? An empirical investigation of the effects of VC portfolio diversification on fund performance." International Entrepreneurship and Management Journal, 10 (1), pp 139-163.

CHANG, S. J. and H. SINGH (1999). "The impact of modes of entry and resource fit on modes of exit by multibusiness firms." Strategic Management Journal 20(11), pp. 1019-1035.

CHARI, M. D. R., S. DEVARAJ, P. DAVID (2008). "Research note - The impact of information technology investments and diversification strategies on firm performance." Management Science, 54 (1), pp. 224-234

CHATTERJEE, S. and J. SINGH (1999). "Are tradeoffs inherent in diversification moves? A simultaneous model for type of diversification and mode of expansion decisions." Management Science 45(1), pp. 2541.

CHATTERJEE, S. and B. WERNERFELT (1991). "The link between resources and type of diversification: Theory and evidence." Strategic Management Journal 12(1), pp. 33-48.

CHEN, L. L. and A. M. L. WANG (2012). "International evidence on industrial diversification and asymmetric information across different ownership structures." Asia Pacific Management Review 17(1), pp. 37-58. 
CHEN, R., M. C. DYBALL, S. WRIGHT (2009). "The link between board composition and corporate diversification in Australian corporations." Corporate Governance: An International Review, 17(2): 208-223.

CHIANG, C. C. (2010). "Product diversification in competitive R\&D-intensive firms: An empirical study of the computer software industry." The Journal of Applied Business Research, 26(1), pp. 99-108.

CHUNG, C. N. and X. LUO (2008). "Human agents, contexts, and institutional change: The decline of family in the leadership of business groups." Organization Science. 19(1). pp, 124-142.

CHUNG, C. N. and X. LUO (2008b). "Institutional logics or agency costs: The influence of corporate governance models on business group restructuring in emerging economies." Organization Science 19(5), pp. 766-784.

de SOUZA VILAS, B. R. (2009). International diversification: entry mode, location decision and performance, (Tesis doctoral) Universidad de Salamanca, Faculty of Economics and Business.

FERNÁNDEZ, M. and I. DÍEZ VIAI (2013). "Effect of firm's resources on international diversification: An application in the Iberian Ham industry." European Management Journal 31(2), 196- 208.

FLETCHER, J. and A. MARSHALL (2005). "An empirical examination of the benefits of international diversification." Journal of International Financial Markets, Institutions and Money. 15, 455-468.

FORCADELL, F. J., B. ALONSO, C., M. GUERRAS, L. A. (2006). Corporate diversification and technological resources dynamics in an organic growth context. In Academy of Management Proceedings. 1, pp. A1-A6.

GALVÁN, V. A. (2009). "Diversification strategies and firm value: causes and consequences, internacional evidence." (Tesis doctoral) Universidad de Salamanca, Faculty of Economics and Business.

GARCIA, S. G. (2003). El gobierno corporativo y las decisiones de crecimiento empresarial: evidencia en las cajas de ahorro españolas, (Tesis doctoral) Universidad de las Palmas de Gran Canaria, Facultad de Ciencias Económicas y Empresariales.
GARY, M. S. (2005). "Implementation strategy and performance outcomes in related diversification." Strategic Management Journal 26(7),pp. 643-664.

GAUR, A. S. and V. KUMAR (2009). "International diversification, business group affiliation and firm performance: Empirical evidence from India." British Journal of Management, 20(2), pp. 172-186.

GEORGE, R. (2007). "Diversification and firm performance: The moderating influence of ownership structure and business group-affiliation." South Asian Journal of Management 14(3), pp. 66-94.

GONG, Y. R., D. P. WANG, Z. MA. (2007). Diversification discount of Chinese listed corporations: Empirical evidence based on agency theory. International Conference on Management Science \& Engineering. ICMSE 2007. IEEE pp. 1322-1327.

GOURLAY, A. and J. SEATON (2004). "The determinants of firm diversification in UK quoted companies." Applied Economics, 36(18), pp. 2059-2071.

GUBBI, S. R., P. S. AULAKH, S. RAY, M. SARKAR AND R. CHITTOOR (2010). Do international acquisitions by emerging-economy firms create shareholder value? The case of Indian firms. Journal of International Business Studies, 41(3), 397-418.

HAI, X. S. and S. W. FU (2011). Research on the effect of diversification strategy on analyst following in Chinese listed companies. International Conference on Management Science \& Engineering (ICMSE,2011). pp. 817-822.

HAUSER, C., J. HOGENACKER, and K. WAGNER. (2013). "International market diversification of innovative European SMEs - What role do various innovation strategies play?". International Journal of Entrepreneurial Venturing 5(3), pp. 310-326.

HERRMANN, P. and D. K. DATTA (2005). "Relationships between top management team characteristics and international diversification: An empirical investigation." British Journal of Management, 16(1), pp. 69-78.

HITT, M. A., R. E. HOSKISSON, and H. KIM (1997). "International diversification: Effects on innovation and firm performance in product-diversified firms." Academy of Management journal 40(4), pp. 767798. 
HOSKISSON, R. E. and M. A. HITT (1990). "Antecedents and performance outcomes of diversification: A review and critique of theoretical perspectives." Journal of Management 16(2), pp. 461-509.

HOSKISSON, R. E., M. A. HITT, R. A. JOHNSON and D. D. MOESEL. (1993). "Construct validity of an objective (entropy) categorical measure of diversification strategy." Strategic Management Journal 14(3), pp. $215-235$.

IBRAHIM, Y. M., A. M. IBRAHIM, and B. KABIR (2009). "Geographic diversification, performance, and the risk profile of UK construction firms." Journal of Engineering, Design and Technology. 7(2), pp. 171-185.

KANG, J. (2013). "The relationship between corporate diversification and corporate social performance." Strategic Management Journal, 34(1), pp. 94-109.

KHANNA, T. and K. PALEPU (2000). "Is group affiliation profitable in emerging markets? An analysis of diversified Indian business groups." The Journal of Finance 55(2), pp. 867-891.

KIM, W. C. and P. HWANG (1992). "Global strategy and multinationals' entry mode choice." Journal of International Business Studies, 23(1), 29-53.

KUPPUSWAMY, V. and B. VILLALONGA (2010). "Does diversification create value in the presence of external financing constraints? Evidence from the 2007-2009 financial crisis." Working paper, Harvard Business School: 10-101.

LEE, K. and X. JIN (2009). "The origins of business groups in China: An empirical testing of the three paths and the three theories." Business History, 51(1), pp. 77-99.

LETEN, B., R. BELDERBOS, and B. VAN LOOY (2007). "Technological diversification, coherence, and performance of firms." Journal of Product Innovation Management, 24(6), pp 567-579.

LICHTENTHALER, E. (2005). "Corporate diversification: identifying new businesses systematically in the diversified firm." Technovation 25(7), pp. 697-709.

LIEN, L. B. and P. G. KLEIN (2013). "Can the survivor principle survive diversification?". Organization Science, 24(5), pp. 1478 - 1494.
LIU, H. Y. and C. W. HSU (2011). "Antecedents and consequences of corporate diversification: A dynamic capabilities perspective." Management Decision 49(9), pp. 1510-1534.

MACKEY, T. B. and J. B. BARNEY (2006). Is there a diversification discount? diversification, payout poli$\mathrm{cy}$, and the value of a firm. Academy of Managment Proceedings 2006, 1. pp. NN1-NN6.

MARINELLI, F. (2011). "The relationship between diversification and firm's performance: is there really a casual relationship." Working Paper IESE Business School - University of Navarra 2011.

MARTíNEZ, A. and R. FERNÁNDEZ (2009). "¿ Qué factores determinan la decisión de diversificar?: El caso de las empresas españolas (1997-2000)." Investigaciones Europeas de Dirección y Economía de la Empresa 15(1), pp. 15-28.

MENDES-DA-SILVA, W., E. L. BLACK, and J. S. MALLETT (2008). "The association between corporate governance and product diversification in Brazilian firms: An empirical study." Corporate Ownership and Control, 5(2), pp. 367-378.

MICHAEL, S. C. (2007). "Can information technology enable profitable diversification? An empirical examination." Journal of Engineering and Technology Management - JET-M 24(3), pp. 167-185.

MILLER, D. J. (2006). "Technological diversity, related diversification, and firm performance." Strategic Management Journal 27(7), pp. 601-619.

MILLER, D. J., M. J. FERN, and L. B. CARDINAL (2007). "The use of knowledge for technological innovation within diversified firms." Academy of Management journal 50(2), pp. 308-326.

MISHRA, A. and M. AKBAR (2007). "Empirical examination of diversification strategies in business groups: Evidence from emerging markets." International Journal of Emerging Markets 2(1), pp. 22-38.

MONTGOMERY, C. A. (1982). "The measurement of firm diversification: Some new empirical evidence." Academy of Management journal 25(2), pp. 299307.

MONTGOMERY, C. A. (1994). "Corporate diversification." The Journal of Economic Perspectives, 8(3), pp. 163-178. 
NACHUM, L. (2004). "Geographic and Industrial Diversification of Developing Country Firms." Journal of Management Studies, 41(2), pp. 273-294.

NAYYAR, P. R. (1992). "On the measurement of corporate diversification strategy: Evidence from large US service firms." Strategic Management Journal 13(3), pp. 219-235.

O'BRIEN, J. P., P. DAVID, T. YOSHIKAWA and A. DELIOS (2014). "How capital structure influences diversification performance: A transaction cost perspective." Strategic Management Journal 35(7), pp. 1013-1031.

PALEPU, K. (1985). "Diversification strategy, profit performance and the entropy measure." Strategic Management Journal 6(3), pp. 239-255.

PARK, K. and S. JANG (2012). "Effect of diversification on firm performance: Application of the entropy measure." International Journal of Hospitality Management 31(1), pp. 218-228.

PARK, K. and S. JANG (2013). "Effects of within-industry diversification and related diversification strategies on firm performance." International Journal of Hospitality Management, 34, pp. 51-60.

PURKAYASTHA, S. (2013). "Diversification Strategy and Firm Performance: Evidence from Indian Manufacturing Firms." Global Business Review, 14(1), pp. 1-23.

RAMANUJAM, V. and P. VARADARAJAN (1989). "Research on corporate diversification: A synthesis." Strategic Management Journal 10(6), pp. 523-551.

RAVICHANDRAN, T., Y. LIU, S. HAN and I. HASAN (2009). "Diversification and firm performance: Exploring the moderating effects of information technology spending." Journal of Management Information Systems, 25(4), pp. 205-240.

RAWLEY, E. and T. S. SIMCOE (2008). Horizontal diversification and vertical contracting: Firm scoe and asset ownership in taxi fleets. Paper presented at 2008 Academy of Management Annual Meeting, L.A, USA.

RíOS, R. D. M. (2012). "Estrategia de diversificación y estructuras de gobierno corporativo: influencia de las oportunidades de crecimiento y el ciclo de vida de la empresa." (Tesis doctoral) Universidad de Salamanca, Departamento de Administración y Economía de la Empresa.
RODRÍGUEZ-DUARTE, A., F. D. SANDULLI, B. MINGUELA-RATA and J. I. LÓPEZ-SÁNCHEZ (2007). "The endogenous relationship between innovation and diversification, and the impact of technological resources on the form of diversification." Research Policy 36(5), pp. 652-664.

RUMELT, R. P. (1974). "Strategy, structure, and economic performance." Harvard University Press, Cambridge. MA.

RUMELT, R. P. (1982). "Diversification strategy and profitability." Strategic Management Journal 3(4): 359-369.

SAMBHARYA, R. B. and J. LEE (2014). "Renewing Dynamic Capabilities Globally: An Empirical Study of the World's Largest MNCs." Management International Review. 54(2), pp 137-169.

SANCHEZ, B. M. J. and B. USERO (2013). "How may the nature of family firms explain the decisions concerning international diversification?" 67(7), pp. 1311-1320.

SÁNCHEZ PEINADO, L., E. SÁNCHEZ PEINADO, and A. ESCRIBÁ ESTEVE (2010). "Factores determinantes de la intención de cambio estratégico: el papel de los equipos directivos." Cuadernos de economía y dirección de la empresa 13(42): 75-112.

SÁNCHEZ, P. L. and B. M. MENGUZZATO (2009). "Antecedents of entry mode choice when diversifying." Industrial Marketing Management 38(8), pp. 971-983.

SANDULLI, F. D., J. FERNANDEZ-MENENDEZ, A. RODRIGUEZ-DUARTE and J. I. LOPEZ-SANCHEZ (2012). "The productivity payoff of information technology in multimarket SMEs." Small Business Economics 39(1), pp. 99-117.

SCHUH, G., M. WELLENSIEK, and J. VON MANGOLDT (2013). "Competence-based diversification: A conceptual approach for evaluating the attractiveness of new market opportunities". Proceedings of PICMET '13: Technology Management for Emerging Technologies. pp. 2344-2352.

SHI, F., Y. WANG, and J. Y. YU (2010). Empirical research of relationship between the level of diversification and corporate performance on China listed company. International Conference on Management Science \& Engineering (17th) 2010. 
SHYU, J. and Y. L. CHEN (2009). "Diversification, performance, and the corporate life cycle." Emerging Markets Finance and Trade. 45(6), pp. 57-68.

SIMMONDS, P. G. (1990). "The combined diversification breadth and mode dimensions and the performance of large diversified firms." Strategic Management Journal 11(5), pp. 399-410.

SINGH, D. A. and A. S. GAUR (2009). "Business group affiliation, firm governance, and firm performance: Evidence from china and india." Corporate Governance: An International Review, 17(4), pp 411-425.

SONG, H. X. and F. S. WANG (2011). Research on the relationship between corporate governance structure and diversification strategy. Management and Service Science (MASS 2011), International Conference, pp 1-4.

STEINER, G. A. (1964). "Why and how to diversify." California Management Review 6(4), pp. 11-18.

TANRIVERDI, H. (2005). "Information technology relatedness, knowledge management capability, and performance of multibusiness firms." MIS Quarterly: Management Information Systems 29(2), pp. 311334.

TANRIVERDI, H. (2006). "Performance effects of information technology synergies in multibusiness firms." MIS quarterly, 30(1), pp.57-77.

TANRIVERDI, H. and C.H. LEE (2008). "Within-industry diversification and firm performance in the presence of network externalities: Evidence from the software industry." Academy of Management journal 51(2), pp. 381-397.

TANRIVERDi, $H$. and N. VENKATRAMAN (2005). "Knowledge relatedness and the performance of multibusiness firms." Strategic Management Journal 26(2), pp. 97-119.

TIHANYI, L., D. A. GRIFFITH, and C. J. RUSSELL (2005). "The effect of cultural distance on entry mode choice, international diversification, and MNE performance: A meta-analysis." Journal of International Business Studies. 36(3), pp. 270-283.

VARADARAJAN, P. R. (1986). "Product diversity and firm performance: An empirical investigation." The Journal of Marketing, pp. 43-57.
VILLALONGA, B. (2004). "Diversification discount or premium? New evidence from the business information tracking series." The Journal of Finance 59(2), pp. 479-506.

VILLASALERO, M. (2013). "Signaling, spillover and learning effects of knowledge flows on division performance within related diversified firms." Journal of Knowledge Management 17(6), pp. 928-942.

WANG, Y. F. and F. YANG (2013). An empirical study on relationship between diversification strategy and research and development investment. The 19th International Conference on Industrial Engineering and Engineering Management, Springer Berlin Heidelberg. pp. 475-484.

WHITE, R. E., R. E. HOSKISSON, D. W. YIU and G. D. BRUTON (2008). "Employment and market innovation in Chinese business group affiliated firms: The role of group control systems." Management and Organization Review. 4(2), pp. 225-256.

WIERSEMA, M. F. and H. P. BOWEN (2008). "Corporate diversification: The impact of foreign competition, industry globalization, and product diversification." Strategic Management Journal, 29(2) 115-132.

WRIGLEY, L. (1970). Divisional autonomy and diversification, (Doctoral dissertation. Harvard Business School, 1970).

YANG, Y., J. J. ZHAO, S. CHEN and H. SUN (2012). Effects of diversified strategic resource configuration on corporate performance: Evidence from listed companies in pharmaceutical and biotech industry in China. 19th International Conference on Management Science \& Engineering, pp. 467 - 474.

YILDIRIM-ÖKTEM, Ö. and B. ÜSDIKEN (2010). "Contingencies versus external pressure: Professionalization in boards of firms affiliated to family business groups in late-industrializing countries." British Journal of Management. 21(1), pp. 115-130.

YIU, D., G. D. BRUTON and Y. LU (2005). "Understanding business group performance in an emerging economy: Acquiring resources and capabilities in order to prosper." Journal of Management Studies, 42(1), pp 183-206. 